\title{
Supercurrent in Long SFFS Junctions with Antiparallel Domain Configuration
}

\author{
Ya. M. Blanter ${ }^{a}$ and F. W. J. Hekking ${ }^{b}$ \\ ${ }^{a}$ Department of NanoScience and DIMES, Delft University of Technology, Lorentzweg 1, 2628 CJ Delft, The Netherlands \\ ${ }^{b}$ Laboratoire de Physique et Modélisation des Milieux Condensés, \\ CNRS \& Université Joseph Fourier, B.P. 166, 38042 Grenoble cedex 9, France
}

(Dated: October 29, 2018)

\begin{abstract}
We calculate the current-phase relation of a long Josephson junction consisting of two ferromagnetic domains with equal, but opposite magnetization $h$, sandwiched between two superconductors. In the clean limit, the current-phase relation is obtained with the help of Eilenberger equation. In general, the supercurrent oscillations are non-sinusoidal and their amplitude decays algebraically when the exchange field is increased. If the two domains have the same size, the amplitude is independent of $h$, due to an exact cancellation of the phases acquired in each ferromagnetic domain. These results change drastically in the presence of disorder. We explicitly study two cases: Fluctuations of the domain size (in the framework of the Eilenberger equation) and impurity scattering (using the Usadel equation). In both cases, the current-phase relation becomes sinusoidal and the amplitude of the supercurrent oscillations is exponentially suppressed with $h$, even if the domains are identical on average.
\end{abstract}

PACS numbers: $74.45 .+\mathrm{c}, 74.50 .+\mathrm{r}$

\section{INTRODUCTION}

Hybrid systems containing superconducting and ferromagnetic elements gained recently a lot of attention due to experimental progress as well as possible applications in magnetoelectronics and quantum information. Theoretical studies are revealing a variety of new features, making these system generators of novel theoretical concepts.

It is a common knowledge that current in hybrid normal metal - superconductor (NS) systems flows by means of Andreev reflections: an electron in $\mathrm{N}$ is reflected from the NS interface as a hole with the opposite charge and velocity. Imagine first that the piece of normal metal is ballistic. An electron at the Fermi surface is reflected as a hole at the Fermi surface, and they propagate in the normal metal with the same phase. If the electron is taken at a finite energy $E$ (counted from the Fermi surface), a momentum mismatch $\delta p=2 E / v_{F}$ between this electron and the reflected hole appears, $v_{F}$ being the Fermi velocity.

Consider now an interface between an ( $s$-wave) superconductor and a ferromagnet. Electron and hole have opposite spin directions, and the exchange field $h$ in the ferromagnet leads to a Zeeman splitting of energies of the two different spin projections. Thus, even an electron and a hole at the Fermi surface acquire the momentum mismatch $2 h / v_{F}$; hence their relative phase grows as $\delta \varphi=2 h x /\left(\hbar v_{F}\right)$, where $x$ is the distance from the interface. This affects phase-sensitive physical quantities like the supercurrent in superconductor - ferromagnet - superconductor (SFS) junctions: It becomes an oscillating function of the thickness $d$ of the ferromagnetic layer, with a period $\hbar v_{F} / 2 h$. If, furthermore, the ferromagnet is diffusive, the oscillating behavior is accompanied by an exponential decay $\propto \exp \left(-(h / \hbar D)^{1 / 2} d\right)$, where $D$ is the diffusion coefficient. Typically, $h$ is much larger than the superconducting gap $\Delta$, and thus the length scales related to the magnetic field are much shorter than the superconducting coherence length $\xi, \hbar v_{F} / \Delta$ and $(\hbar D / \Delta)^{1 / 2}$ in the clean and diffusive case, respectively. In other words, the proximity effect is suppressed in the ferromagnet.

This qualitative discussion suggests that the main effect observed in SFS contacts is oscillations of the supercurrent with the thickness of the ferromagnetic layer - the transition from a so-called 0-state (energy of the contact is minimum for zero phase difference between the superconductors) to a $\pi$-state (energy is minimum for a phase difference $\pi$ ). This topic was at the focus of attention since the early exploration of the field [1]. Theoretically, the $\pi$-state was predicted in a variety of SFS junctions: Ballistic 2, 3, 4, 5], short diffusive 6, 7], long diffusive [5, 6, 8], ferromagnetic insulating barrier 1, 9, 10], ballistic [11, 12, 13] and diffusive [5, 14, 15, 16, 17, 18] junctions with a barrier separating two ferromagnetic layers, and ballistic 19 and diffusive 18 with two tunnel barriers. The transition to the $\pi$-state was recently observed experimentally in SFS junctions 20, 21, 22, 23]. All these observations are limited to small thickness of ferromagnetic layer(s), $d \lesssim(\hbar D / h)^{1 / 2}$. For thicker layers, supercurrent does not exist.

In this situation, it is useful to understand how one can enhance the proximity effect. Several options have been recently discussed in the literature. First, the above qualitative argument assumes that the pairing between an electron and a hole participating in the Andreev reflection is singlet - they have opposite spin projections. Obviously, if the superconductor allows for a non-trivial symmetry of the order parameter, this needs not be the case, and triplet pairing between an electron and a hole with the same spin projection can arise. Since tripletpaired electron and hole at the Fermi surface have no momentum difference, they can propagate with the same 
phase and enhance the proximity effect. Coupling of two $d$-wave superconductors via a ferromagnetic layer has been considered in Ref. 24. Moreover, triplet pairing can even appear in a contact of an $s$-wave superconductor and a ferromagnet, provided the magnetization in the latter is non-uniform [25, 26, 27]. In this case, the proximity effect survives at the same distance $\xi$ from the interface as in non-magnetic metals. Indeed, the supercurrent in SFS junctions with non-uniform magnetization is considerably enhanced [5]. We also mention that the supercurrent in a long diffusive SFS junction is exponentially suppressed only on average; phenomena related to the proximity effect still occur in such a junction as a result of mesoscopic fluctuations around average quantities [28]. Finally, if the ferromagnetic layer is split into domains, the coherence can be preserved if an electron and a hole propagate between the superconducting electrodes along the two sides of a domain wall [29].

In this Article, we explore a different way to enhance the supercurrent in SFS junctions. Imagine first that the junction is ballistic and the ferromagnetic layer consists of two domains with opposite directions of the magnetization, as shown in Fig. 1. Triplet pairing is not generated in this geometry. Consider an electron and an Andreevreflected hole propagating from left to right between the superconducting electrodes. They first acquire the relative phase $\delta \varphi_{1}=2 h x_{1} /\left(\hbar v_{F}\right), x_{1}$ being the distance traversed in the first ferromagnetic layer. However, in the second layer the exchange field has the opposite sign, and the phase gain $\delta \varphi_{2}=-2 h x_{2} /\left(\hbar v_{F}\right)$ partially compensates $\delta \varphi_{1}$. For $x_{1}=x_{2}$ we have full compensation: The ferromagnetic bilayer behaves as a piece of normal (not ferromagnetic) metal, and the proximity effect is fully restored. Indeed, previous studies of SFS contacts where the two ferromagnetic domains were separated by a barrier, found that the supercurrent in the antiparallel domain configuration is enhanced with respect to the parallel one 11, 13, 15, 18]. If the domains are identical, there is no transition to the $\pi$-sate in the antiparallel configuration.

Below, we consider such a situation quantitatively. Section II treats a ballistic SFFS junction with two ferromagnetic domains parallel to the superconducting interfaces. We show that this system behaves as a ballistic SFS junction with an effective exchange field. If the widths of the two domains are the same, this effective field vanishes. In the next two Sections, we study the effect of disorder in the same system and show that supercurrent in diffusive SFFS junctions decays exponentially with their width, similarly to SFS contacts without domains. We consider long junctions, $d \gg \xi$, and assume that the superconducting electrodes do not influence the magnetic structure of the contact.

\section{CLEAN SFFS CONTACT}

We consider first a system of two clean ferromagnetic strips [30] with the thicknesses $d_{1}$ and $d_{2}$ and antiparallel orientations located between two superconductors (Fig. 1). The dynamics of quasiparticles in this system are described by the Eilenberger equation,

$$
-i v_{F} \boldsymbol{n} \nabla \breve{g}_{\sigma}(\boldsymbol{r}, \boldsymbol{n})=\left[(i \omega \mp h \sigma) \breve{\tau}_{3}+\breve{\Delta}, \breve{g}_{\sigma}(\boldsymbol{r}, \boldsymbol{n})\right]_{-} .
$$

Here the semi-classical Green's function $\breve{g}_{\sigma}$ is a matrix in Nambu space,

$$
\breve{g}_{\sigma}=\left(\begin{array}{rr}
g_{\sigma} & f_{\sigma} \\
f_{\sigma}^{+} & -g_{\sigma}
\end{array}\right)
$$

which describes the singlet pairing (the triplet component is not generated in our geometry), and the spin index $\sigma= \pm 1$. The exchange field $h$ is zero in the superconducting banks, and has antiparallel orientations in the ferromagnets: The upper/lower signs in Eq. (1) corresponds to the left/right ferromagnet $(h>0)$. To stay in the framework of the semi-classical consideration, we have assumed that the Zeeman splitting $h$ is much weaker than the Fermi energy, but can be arbitrary in comparison with the superconducting gap $\Delta$. We put $\hbar=1$; it will be restored in the final results.

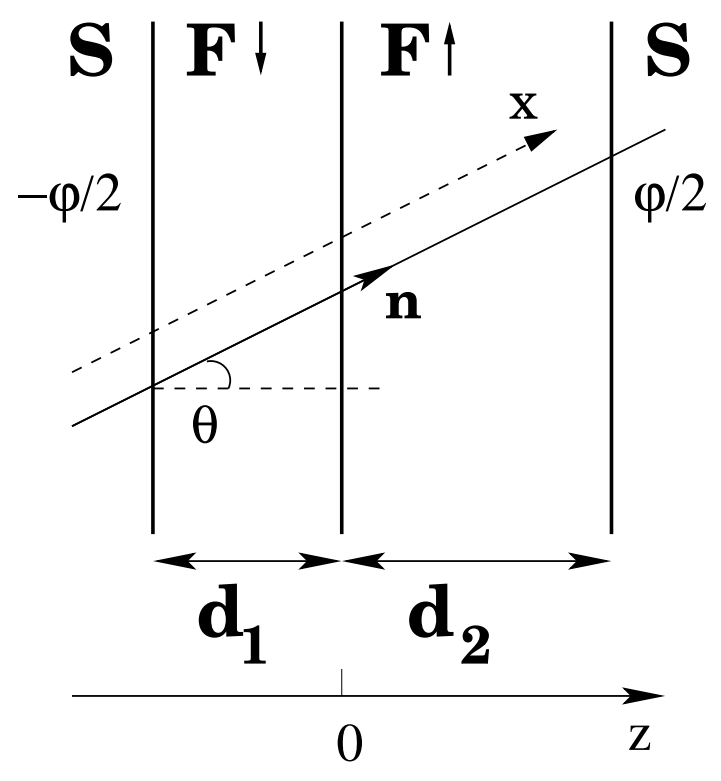

FIG. 1: Setup with two ferromagnetic domains with antiparallel configurations.

In this Article, we consider the case of a long contact: The thicknesses of both ferromagnetic layers are much larger than the superconducting coherence length, $d_{1,2} \gg$ 
$\hbar v_{F} / \Delta$. Then the matrix $\breve{\Delta}$ can be taken in a piecewise approximation: It is zero in both ferromagnets, and

$$
\breve{\Delta}=\left(\begin{array}{lr}
0 & \Delta e^{i \chi} \\
-\Delta e^{-i \chi} & 0
\end{array}\right)
$$

in the superconductors. Here $\chi=-\varphi / 2$ and $\chi=\varphi / 2$ in the left and the right superconducting bank, respectively.

In the bulk superconductor far from the contacts the Green's function is isotropic and equals for $|\omega|<\Delta$

$$
\breve{g}_{\sigma}^{b u l k}=\frac{1}{\sqrt{\Delta^{2}+\omega^{2}}}\left(\begin{array}{lr}
\omega & -i \Delta e^{i \chi} \\
i \Delta e^{-i \chi} & -\omega
\end{array}\right) .
$$

In addition, the Green's function and its derivative must be continuous at each interface.

We introduce the coordinate $x$ parallel to $\boldsymbol{n}$ and directed from left to right. Let us choose $x=0$ at the boundary of the left superconductor; then $x=d_{1} / \cos \theta$ at the interface of the two ferromagnets, and $x=\left(d_{1}+\right.$ $\left.d_{2}\right) / \cos \theta$ at the boundary of the right superconductor. The quasiparticles in the clean system move along a straight line (Fig. 11). It follows from Eq. (11) that the normal component $g_{\sigma}(\boldsymbol{r}, \boldsymbol{n})$ is constant along the trajectory inside the ferromagnets. The calculation gives

$$
g_{\sigma}(\boldsymbol{n})=\frac{\sqrt{\Delta^{2}+\omega^{2}} \sin \alpha+i \omega(1+\cos \alpha)}{\omega \sin \alpha+i \sqrt{\Delta^{2}+\omega^{2}}(1+\cos \alpha)},
$$

where the phase $\alpha$ accumulated along the trajectory is

$$
\alpha=\frac{2 i \omega}{v_{F}} \frac{d_{1}+d_{2}}{\cos \theta}+\frac{2 h \sigma}{v_{F}} \frac{d_{1}-d_{2}}{\cos \theta}-n_{x} \varphi, \quad n_{x}= \pm 1 .
$$

The supercurrent density is expressed as follows,

$$
j=-i \pi e v_{F} \nu \sum_{\sigma} T \sum_{\omega} \int d \boldsymbol{n} g_{\boldsymbol{n}} \boldsymbol{n},
$$

where $\nu$ is the density of states. For $h=0$ Eq. (5) gives the supercurrent of a long clean SNS (non-ferromagnetic) junction, as considered in Ref. 31, which we follow in the general case. The expression is even in $\omega$; for zero temperature (case of interest here) the summation can be replaced by an integration over frequencies. We subsequently introduce a new integration variable $\omega=\Delta \sinh u$ and arrive at the intermediate expression

$$
\begin{aligned}
& j=2 e v_{F} \nu \Delta \sum_{\sigma} \int_{0}^{\infty} d u \cosh u \int_{0}^{\pi / 2} d \theta \cos \theta \\
& \times \operatorname{Im} \tanh \left[u+\Delta \sinh u \frac{d_{1}+d_{2}}{v_{F} \cos \theta}+i h \sigma \frac{d_{1}-d_{2}}{v_{F} \cos \theta}+i \frac{\varphi}{2}\right] .
\end{aligned}
$$

For long contacts, $\Delta d_{1,2} \gg \hbar v_{F}$, the first term in the argument of the hyperbolic tangent can be disregarded. Using the identity

$$
\operatorname{Im} \tanh y=2 \sum_{k=1}^{\infty}(-1)^{k} \operatorname{Im} e^{-2 k y},
$$

we obtain the final expression for the supercurrent,

$$
\begin{aligned}
j & =\frac{4 e v_{F}^{2} \nu \hbar}{d_{1}+d_{2}} \sum_{k=1}^{\infty} \frac{(-1)^{k+1}}{k} \sin k \varphi \\
& \times \int_{1}^{\infty} \frac{d x}{x^{3} \sqrt{x^{2}-1}} \cos \frac{2 k h\left(d_{1}-d_{2}\right) x}{v_{F} \hbar} .
\end{aligned}
$$

For $h=0$, we return to the clean long SNS contact,

$$
j=j_{0} \sum_{k=1}^{\infty} \frac{(-1)^{k+1}}{k} \sin k \varphi,
$$

where $j_{0}=\pi e v_{F}^{2} \nu \hbar /\left(d_{1}+d_{2}\right)$. This describes the wellknown sawtooth current-phase relation found earlier in Ref. 32 .

For strong magnetic fields, $h \gg \hbar v_{F} /\left|d_{1}-d_{2}\right|$, the integral over $d x$ in Eq. (7), which corresponds to summing over all possible trajectories in the ferromagnets can be calculated in the saddle-point approximation. As a result we find the current-phase relation

$$
\begin{aligned}
j & =2 j_{0} \sqrt{\frac{v_{F} \hbar}{\pi h\left|d_{1}-d_{2}\right|}} \sum_{k=1}^{\infty} \frac{(-1)^{k+1}}{k^{3 / 2}} \\
& \times \cos \left(\frac{2 k h\left|d_{1}-d_{2}\right|}{v_{F} \hbar}+\frac{\pi}{4}\right) \sin k \varphi .
\end{aligned}
$$

We note, first of all, that the amplitude of the supercurrent oscillations as a function of $\varphi$ decreases algebraically with the exchange field, as $\sqrt{\hbar v_{F} / h\left|d_{1}-d_{2}\right|}$. This is a direct consequence of the fact that we summed over all possible trajectories, and hence averaged over the different phases acquired during propagation in the ferromagnetic domains along these trajectories. Secondly, as far as the phase-dependence of the supercurrent is concerned, it is in general neither sinusoidal, nor saw-tooth-like. In Fig. 2] we plot $j(\varphi)$ for various values of $h\left|d_{1}-d_{2}\right| / \hbar v_{F} \sim$ 10 , such that the saddle-point approximation is reasonable. We see that, as a function of the exchange field, the supercurrent changes sign at a given phase difference. Thus, depending on the parameter $h\left|d_{1}-d_{2}\right| / \hbar v_{F}$, the junction either favors a 0 -state or a $\pi$-state. We finally note that for $d_{2}=0$, Eqs. (7) and (9) give the supercurrent for a (single-domain) clean long SFS junction. This is, to our knowledge, a new result as well. It implies in particular that a clean SFS junction can also be a $\pi$-junction, in accordance with previous results for different types of SFS hybrid structures.

The important conclusion for the general case is that for a two-domain contact the result is exactly the same as for a SFS junction with the thickness $d_{1}+d_{2}$ and the effective exchange field $h_{e f}=h\left|d_{1}-d_{2}\right| /\left(d_{1}+d_{2}\right)$. In particular, if the thicknesses are the same, $d_{1}=d_{2}$, the magnetic field drops out - we obtain the sawtooth current-phase relation (8) like for a SNS contact. In the language of Eilenberger equations, this statement is obvious: Indeed, the only quantity sensitive to the magnetic field is the phase $\alpha$ accumulated along the trajectory. 


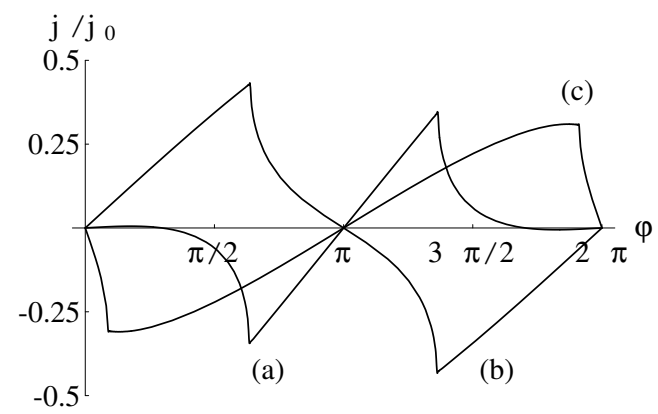

FIG. 2: Supercurrent $j$ in units of $j_{0}$ as a function of $\varphi$ for various values of $h: h\left|d_{1}-d_{2}\right| / \hbar v_{F}=10.0$ (a), 12.0 (b), and 14.0 (c).

Since each trajectory is a straight line, each layer contributes with a weight proportional to its thickness and with the sign depending on the direction of the exchange field. This result is readily generalized to the case of many ferromagnetic layers in the antiparallel configuration [33].

\section{DISORDER AVERAGING}

Now we discuss how our two main observations for the supercurrent - power-law decay with magnetic field and the independence on the magnetic field in the symmetric case $d_{1}=d_{2}$ - react to the presence of disorder. Before performing this difficult task in Section IV by solving the Usadel equations, we try to use an easy way to understand the effect of impurities in this Section. We introduce randomness in the thicknesses of the layers (surface randomness). This simple and transparent calculation provides us with results which are clear qualitative predictions to be compared with the conclusions extracted from a more complicated analysis of the Usadel equations.

We start from Eq. (7), and imagine that the interfaces as presented in Fig. 1 are not straight, but exhibit small fluctuations in position. Since there is no scattering at the interfaces, the only effect of such fluctuations is that the thicknesses of the layers become random variables, and the supercurrent (7) must be averaged with respect to this randomness. Let us take a Gaussian distribution for the difference $d_{1}-d_{2}$,

$$
P\left(d_{1}-d_{2}\right)=\frac{1}{\sqrt{\pi a}} \exp \left(-\frac{\left(d_{1}-d_{2}-\bar{d}_{1}+\bar{d}_{2}\right)^{2}}{a^{2}}\right)
$$

where $a \ll \bar{d}_{1}, \bar{d}_{2}$ has the meaning of a typical scale of the interface fluctuations, and $\bar{d}_{i}$ are the averaged values both thicknesses. Averaging Eq. (17), we obtain

$$
\begin{aligned}
\bar{j} & =\frac{4 \bar{j}_{0}}{\pi} \sum_{k=1}^{\infty} \frac{(-1)^{k+1}}{k} \sin k \varphi \int_{1}^{\infty} \frac{d x}{x^{3} \sqrt{x^{2}-1}} \\
& \times \exp \left(-\frac{k^{2} h^{2} a^{2} x^{2}}{v_{F}^{2} \hbar^{2}}\right) \cos \frac{2 k h\left(\bar{d}_{1}-\bar{d}_{2}\right) x}{v_{F} \hbar}
\end{aligned}
$$

where $\bar{j}_{0}=\pi e v_{F}^{2} \nu \hbar /\left(\bar{d}_{1}+\bar{d}_{2}\right)$. In strong fields, $h \gg$ $\hbar v_{F} / a \gg \hbar v_{F} /\left(\bar{d}_{1}-\bar{d}_{2}\right)$, the integral is calculated in the saddle-point approximation, and only the term with $k=$ 1 survives,

$$
\begin{aligned}
\bar{j} & =-2 \bar{j}_{0} \sqrt{\frac{v_{F} \hbar}{\pi h\left|\bar{d}_{1}-\bar{d}_{2}\right|}} \exp \left(-\frac{h^{2} a^{2}}{v_{F}^{2} \hbar^{2}}\right) \\
& \times \cos \left(\frac{2 h\left(\bar{d}_{1}-\bar{d}_{2}\right)}{v_{F} \hbar}+\frac{\pi}{4}\right) \sin \varphi .
\end{aligned}
$$

Thus, the averaging procedure brings out two, qualitatively new features: (i) at high fields, the current-phase relation becomes sinusoidal; (ii) the amplitude of the supercurrent oscillations decays exponentially, rather than algebraically with $h$. In addition, the exchange field still modulates the phase of the oscillations, and can drive the contact to a $\pi$-state. The property (i) stems from phaseaveraging over diffusive trajectories and is a common feature of all long disordered SNS junctions ( $c$ f Ref. 34).

Eq. (12) does not apply to the symmetric case $\bar{d}_{1}=\bar{d}_{2}$. In this situation, for $h \gg v_{F} / a$, we have

$$
\bar{j}=-\frac{2 \bar{j}_{0}}{\sqrt{\pi}} \frac{v_{F} \hbar}{h a} \exp \left(-\frac{h^{2} a^{2}}{v_{F}^{2} \hbar^{2}}\right) \sin \varphi .
$$

We see that even for the symmetric case the exponential dependence on magnetic field persists. It reflects the fact that a quasiparticle moving along a single trajectory spends in general unequal times in both layers, and thus the contribution of each trajectory is magnetic field dependent. However, there is no additional oscillating factor due to the magnetic field: a symmetric junction is never in the $\pi$-state. These features are confirmed qualitatively in the next Section, where we analyze the behavior of a symmetric diffusive SFFS junction, using the Usadel equations.

\section{DISORDERED SFFS CONTACT FROM USADEL EQUATIONS}

We now consider a diffusive SFFS junction in the symmetric case $d_{1}=d_{2}=d / 2$. The junction is again assumed to be long, $d \gg(\hbar D / \Delta)^{1 / 2}$, with $D$ being the diffusion coefficient.

If the exchange magnetic energy does not exceed the inverse elastic scattering time, $h \ll \hbar / \tau$, the Green's function is almost isotropic, and the system can be described by Usadel equations,

$$
\begin{aligned}
& D \partial_{z}\left[F_{\sigma} \partial_{z} F_{\sigma}^{+}-F_{\sigma}^{+} \partial_{z} F_{\sigma}\right]=2\left(\tilde{\Delta} F_{\sigma}^{+}-\tilde{\Delta}^{*} F_{\sigma}\right), \\
& D \partial_{z}\left[G_{\sigma} \partial_{z} F_{\sigma}-F_{\sigma} \partial_{z} G_{\sigma}\right]=2(\omega \pm i h \sigma) F_{\sigma}-2 \tilde{\Delta} G_{\sigma}
\end{aligned}
$$


with the constraint $G_{\sigma}^{2}+F_{\sigma} F_{\sigma}^{+}=1$. Here, as usual [34],

$$
\breve{G}_{\sigma}(\boldsymbol{r})=\int d \boldsymbol{n} \breve{g}_{\sigma}(\boldsymbol{r}, \boldsymbol{n})
$$

and it actually only depends on the distance $z$ from the ferromagnet-ferromagnet interface (Fig. 11). The upper/lower signs describe the regions $-d / 2<z<0$ and $0<z<d / 2$, respectively, and $\tilde{\Delta}=i \Delta \exp (i \chi)$ in the superconductors. In the following, we suppress the spin index $\sigma$ where it does not lead to ambiguities.

Following Ref. 31, we solve the constraint by introducing two complex-valued fields $\theta$ and $\eta$,

$$
G=\cos \theta, \quad F=\sin \theta e^{i \eta}, \quad F^{+}=\sin \theta e^{-i \eta} .
$$

The equation for $\eta$ in the ferromagnets becomes

$$
\partial_{z}\left(\eta^{\prime} \sin ^{2} \theta\right)=0
$$

with the boundary conditions $\eta( \pm d / 2)=\mp \varphi / 2$. The first integral yields

$$
\eta^{\prime}=\frac{I}{\sin ^{2} \theta} .
$$

where $I$ is an unknown constant. The current is expressed via this constant,

$$
\begin{aligned}
j & =\frac{i \pi e D \nu}{2} \sum_{\sigma} T \sum_{\omega}\left[F_{\sigma} \partial_{z} F_{\sigma}^{+}-F_{\sigma}^{+} \partial_{z} F_{\sigma}\right] \\
& =\pi e D \nu \sum_{\sigma} T \sum_{\omega} I_{\sigma} .
\end{aligned}
$$

To ensure the current conservation, $I$ must be the same in both ferromagnetic layers. It is important, however, that we do not assume that the current is conserving - it follows naturally from the consistency of our solution.

Using Eq. (16), we also write the equation for $\theta$ in ferromagnets,

$$
D \theta^{\prime \prime}=D I^{2} \frac{\cos \theta}{\sin ^{3} \theta}+2(\omega \pm i h \sigma) \sin \theta,
$$

with the first integral

$$
D \theta^{\prime 2}=-\frac{D I^{2}}{\sin ^{2} \theta}-4(\omega \pm i h \sigma) \sin \theta+\text { const. }
$$

Now, for the long junctions, the boundary conditions for $\theta$ at $z= \pm d / 2$ are essentially the same they would be at the interface between a semi-infinite superconductor and a semi-infinite ferromagnet. To find these boundary conditions, we write the corresponding equation for the superconductors,

$$
D \theta^{2}=-4 \omega \sin \theta-4 \Delta \cos \theta+\text { const, }
$$

Taking into account that in the bulk superconductor $\theta=$ $\pi / 2$, in the bulk ferromagnet $\theta=0$, and requiring the continuity of $\theta$ and $\theta^{\prime}$ at the interface, we obtain the following boundary conditions,

$$
\omega \pm i h \sigma(1-\cos \theta)+\Delta(1-\sin \theta)+\frac{I^{2}}{\sin ^{2} \theta}=0
$$

at $z=\mp d / 2$. Although our equations describe the behavior of an SFFS junction for an arbitrary relation between $h$ and $\Delta$, we concentrate in the following on the case $T, h \ll \Delta$. As we show below, in this situation the current $I$ is exponentially small, and the boundary condition for $\theta$ reduce to $\theta(z= \pm d / 2)=\pi / 2$.

Since the Usadel equations posses obvious symmetries $\theta_{\sigma}(\omega)=\theta_{-\sigma}(\omega)+\pi, \eta_{\sigma}(\omega)=\eta_{-\sigma}(\omega)+\pi$, in the sequel we only consider $\omega>0$.

The field $\theta$ must rapidly decay away from superconductors and stay exponentially small within the ferromagnets. We start first solving Eq. (20) at $z \ll d$, where $\theta \ll 1$, and the trigonometric functions can be expanded. Then Eq. (20) can be integrated. The solution is too cumbersome to be written down here, its asymptotics for $|z| \rightarrow \infty$ are

$$
\begin{aligned}
\theta & =\frac{1}{2} \sqrt{\left[\theta_{0}+\sqrt{\frac{D \gamma^{2}}{2(\omega \pm i h \sigma)}}\right]^{2}+\frac{D I^{2}}{2 \theta_{0}^{2}(\omega \pm i h \sigma)}} \\
& \times \exp \left(\sqrt{\frac{2}{D}}(\alpha+i \beta \sigma)|z|\right)
\end{aligned}
$$

with the notations $\theta_{0}=\theta(z=0), \gamma=\theta^{\prime}(z=0)$, and

$$
\alpha=\frac{1}{\sqrt{2}} \sqrt{\sqrt{\omega^{2}+h^{2}}+\omega} ; \quad \beta= \pm \frac{1}{\sqrt{2}} \sqrt{\sqrt{\omega^{2}+h^{2}}-\omega} .
$$

Next, we solve Eq. (20) close to the interfaces, $\mid z-$ $d / 2 \mid \ll d$. We assume that $I / \theta_{0}, \gamma$ are both exponentially small (to be checked later) and obtain

$$
\tan \frac{\theta}{4}=\tan \frac{\pi}{8} \exp \left(\sqrt{\frac{2}{D}}(\alpha+i \beta \sigma)\left(|z|-\frac{d}{2}\right)\right) .
$$

Far from the interface, $\theta \ll 1$, the solution becomes exponential. Matching the exponential asymptotics of Eqs. (21) and (22), we find the condition

$$
\begin{aligned}
& {\left[\theta_{0}^{2} \mp \sqrt{\frac{D \gamma^{2}}{2(\omega \pm i h \sigma)}}\right]^{2}+\frac{D I^{2}}{2 \theta_{0}^{2}(\omega \pm i h \sigma)}} \\
& =64 \tan ^{2} \frac{\pi}{8} \exp \left(-\sqrt{\frac{2}{D}}(\alpha+i \beta \sigma) d\right) .
\end{aligned}
$$

We now integrate Eq. (17). Since $\theta(x)$ grows exponentially away from $x=0$, the sine in the denominator can be replaced by its argument. We then find

$$
\sqrt{\frac{D I^{2}}{2(\omega \pm i h \sigma)}}=\theta_{0}\left(\theta_{0} \mp \sqrt{\frac{D \gamma^{2}}{2(\omega+i h \sigma)}}\right) \tan \left(\frac{\varphi}{2} \pm \eta_{0}\right)
$$


with $\eta_{0}=\eta(0)$. We proceed by calculating the four quantities $I, \theta_{0}, \eta_{0}$, and $\gamma$. The result is

$$
\begin{aligned}
I & =\frac{64(\sqrt{2}-1)^{2}}{\sqrt{D}} \sqrt{\frac{\omega^{2}+h^{2}}{\sqrt{\omega^{2}+h^{2}}+\omega}} \\
& \times \exp \left(-\sqrt{\frac{2}{D}} \alpha d\right) \sin \varphi .
\end{aligned}
$$

Note that $I$ does not depend on $\sigma$. It can be easily checked that $I / \theta_{0}$ and $\gamma$ are exponentially small, which justifies the approximations we have made to arrive at Eq. (25).

Now we calculate the supercurrent according to Eq. (18). For high temperatures $T \gg \hbar D / d^{2}, h$ only the term with $\omega=\pi T$ is important, and we obtain

$$
\begin{aligned}
j & =\sqrt{2} j_{0, \operatorname{diff}}\left(\frac{\pi k_{B} T d^{2}}{\hbar D}\right)^{3 / 2} \\
& \times \exp \left(-\frac{d}{\sqrt{\hbar D}} \sqrt{\pi T+\sqrt{h^{2}+\pi^{2} T^{2}}}\right) \sin \varphi
\end{aligned}
$$

where we introduced $j_{0, \text { diff }}=128(\sqrt{2}-1)^{2} e \nu \hbar D^{2} / d^{3}$. In high magnetic fields $h \gg T, \hbar D / d^{2}$ the terms with $\omega<h$ contribute,

$$
j=j_{0, \text { diff }}\left(\frac{h d^{2}}{\hbar D}\right)^{3 / 2} \exp \left(-\sqrt{\frac{h}{\hbar D}} d\right) \sin \varphi .
$$

We note the two main features of the solution in the diffusive case. First, the current-phase relation is sinusoidal. This corresponds to the result for the long diffusive SNS contact [34]. Then, the supercurrent decays exponentially with magnetic field, in contrast to the power-law decay in the clean case.

Similarly, we can treat a single-layer SFS junction of a thickness $d$. The result for $h \gg \hbar D / d^{2}, T$ reads

$$
\begin{aligned}
j & =j_{0, \operatorname{diff}}\left(\frac{h d^{2}}{\hbar D}\right)^{3 / 2} \\
& \times \exp \left(-\sqrt{\frac{h}{\hbar D}} d\right) \sin \left(\sqrt{\frac{h}{\hbar D}} d\right) \sin \varphi .
\end{aligned}
$$

Thus, comparing Eq. (28) with Eq. (27) we see that a long diffusive SFS contact can be a $\pi$-junction, depending on the thickness of the ferromagnet, whereas a similar symmetric SFFS contact with anti-parallel configuration of the domains is not a $\pi$-junction.

\section{DISCUSSION}

We considered the behavior of the supercurrent in long SFS junctions. We obtained new expressions for single-domain ballistic and diffusive contacts and confirmed that the 0 to $\pi$ transition can be induced in these systems. However, our main focus is on the situation when the ferromagnetic region is split into two ferromagnetic domains with equal but opposite magnetization. In the ballistic case, this system behaves as a singledomain SFS junction, with the effective exchange field $h_{e f}=h\left|d_{1}-d_{2}\right| /\left(d_{1}+d_{2}\right)$. Such a system exhibits a non-sinusoidal current-phase relation, and a power-law decay of the supercurrent with thickness and exchange field. If the thicknesses of the both domains are the same, the effective field vanishes. Disorder, considered both as geometrical fluctuations of the thickness, or randomly positioned impurities, restores exponential decay and sinusoidal phase dependence of the supercurrent. A system with two domains of the same width is never in the $\pi$-state.

To obtain these results, we made a number of simplifying assumptions. The superconductor-ferromagnet interfaces, as well as the boundary between the two ferromagnetic domains, are assumed to be ideal (no scattering) and sharp. This can be realized in multilayered structures, where the ferromagnetic layers can be artificially constructed and kept very clean. Another, more attractive option, is real ferromagnetic domains. A domain wall has a finite width, typically of order of the mean free path, or wider. This induces reflection of electrons from the domain wall, and additionally generates the triplet pairing between electrons and holes. These factors need to be taken into account for a quantitative comparison between theory and experiment. However, we do not expect them to add qualitatively new features into the picture we presented.

\section{Acknowledgments}

We thank J. Aarts, E. V. Bezuglyi, Yu. M. Galperin, A. A. Golubov, W. Guichard, and Yu. V. Nazarov for useful discussions. FH acknowledges the hospitality of Delft University of Technology. This work was supported by the Netherlands Foundation for Fundamental Research on Matter (FOM) and by Institut Universitaire de France.
[1] L. N. Bulaevskii, V. V. Kuzii, and A. A. Sobyanin, Pis'ma Zh. Éksp. Teor. Fiz. 25, 314 (1977) [JETP Lett. 25, 290 (1977)].

[2] A. I. Buzdin, L. N. Bulaevskii, and S. V. Panyukov,
Pis'ma Zh. Éksp. Teor. Fiz. 35, 147 (1982) [JETP Lett. 35, 178 (1982)].

[3] L. Dobrosavljević-Grujić, R. Zikić, and Z. Radović, Physica C 331, 254 (2000). 
[4] N. M. Chtchelkatchev, W. Belzig, Yu. V. Nazarov, and C. Bruder, Pis'ma Zh. Éksp. Teor. Fiz. 74, 357 (2001) [JETP Lett. 74, 323 (2001)].

[5] F. S. Bergeret, A. F. Volkov, and K. B. Efetov, Phys. Rev. B 64, 134506 (2001).

[6] A. I. Buzdin and M. Yu. Kupriyanov, Pis'ma Zh. Éksp. Teor. Fiz. 53, 308 (1991) [JETP Lett. 53, 321 (1991)]; A. I. Buzdin, B. Vujičić, and M. Yu. Kupriyanov, Zh. Éksp. Teor. Fiz. 101, 231 (1992) [Sov. Phys. JETP 74, 124 (1992)].

[7] E. A. Demler, G. B. Arnold, and M. R. Beasley, Phys. Rev. B 55, 15174 (1997).

[8] T. T. Heikkilä, F. K. Wilhelm, and G. Schön, Europhys. Lett. 51, 434 (2000).

[9] Y. Tanaka and S. Kashiwaya, Physica C 274, 357 (1997).

[10] M. Fogelström, Phys. Rev. B 62, 11812 (2000); J. C. Cuevas and M. Fogelström, Phys. Rev. B 64, 104502 (2001).

[11] F. S. Bergeret, A. F. Volkov, and K. B. Efetov, Phys. Rev. Lett. 86, 3140 (2001).

[12] Yu. S. Barash and I. V. Bobkova, Phys. Rev. B 65, 144502 (2002).

[13] Yu. S. Barash, I. V. Bobkova, and T. Kopp, Phys. Rev. B 66, 140503 (2002).

[14] E. A. Koshina and V. N. Krivoruchko, Pis'ma Zh. Éksp. Teor. Fiz. 71, 182 (2000) [JETP Lett. 71, 123 (2000)]; E. Koshina and V. Krivoruchko, Phys. Rev. B 63, 224515 (2001).

[15] V. N. Krivoruchko and E. A. Koshina, Phys. Rev. B 64, 172511 (2001).

[16] V N. Krivoruchko and R. V. Petryuk, Phys. Rev. B 66, 134520 (2002).

[17] A. A. Golubov, M. Yu. Kupriyanov, and Ya. V. Fominov, Pis'ma Zh. Éksp. Teor. Fiz. 75, 223 (2002) [JETP Lett. 75, 190 (2002)].

[18] A. A. Golubov, M. Yu. Kupriyanov, and Ya. V. Fominov, Pis'ma Zh. Éksp. Teor. Fiz. 75, 709 (2002) [JETP Lett. 75, 588 (2002)].

[19] Z. Radović, N. Lazarides, and N. Flytzanis, cond-mat/0305437
[20] V. V. Ryazanov, V. A. Oboznov, A. Yu. Rusanov, A. V. Veretennikov, A. A. Golubov, and J. Aarts, Phys. Rev. Lett. 86, 2427 (2001).

[21] T. Kontos, M. Aprili, J. Lesueur, F. Genêt, B. Stephanidis, and R. Boursier, Phys. Rev. Lett. 89, 137007 (2002).

[22] W. Guichard, M. Aprili, O. Bourgeois, T. Kontos, J. Lesueur, and P. Gandit, Phys. Rev. Lett. 90, 167001 (2003).

[23] Y. Blum, A. Tsukernik, M. Karpovski, and A. Palevski, cond-mat/0203408

[24] Z. Radović, L. Dobrosavljević-Grujić, and B. Vujičić, Phys. Rev. B 60, 6844 (1999).

[25] F. S. Bergeret, K. B. Efetov, and A. I. Larkin, Phys. Rev. B 62, 11872 (2000).

[26] F. S. Bergeret, A. F. Volkov, and K. B. Efetov, Phys. Rev. Lett. 86, 4096 (2001); Phys. Rev. B 65, 134505 (2002).

[27] A. Kadigrobov, R. I. Shekhter, and M. Jonson, Europhys. Lett. 54, 394 (2001).

[28] A. Yu. Zyuzin, B. Spivak, and M. Hruška, cond-mat/0204123

[29] R. Melin, J. Phys. Cond. Matter 13, 6445 (2001); N. M. Chtchelkatchev and I. S. Burmistrov, cond-mat/0303014

[30] Generalization to three dimensions (planar contact) is straightforward and the results are qualitatively the same.

[31] A. V. Svidzinskii, Spatially Non-homogeneous Problems of Superconductivity, Nauka, Moscow (1982).

[32] C. Ishii, Prog. Theor. Phys. 44, 1525 (1970); J. Bardeen and J. L. Johnson, Phys. Rev. B 5, 72 (1972); A. I. Bezuglyi, I. O. Kulik, and Yu. N. Mitsai, Fiz. Nizkikh Temp. 1, 57 (1975) [Sov. J. Low Temp. Phys. 1, 27 (1975)].

[33] In this case, the magnetic fields in all the layers need to be collinear, otherwise triplet pairing is generated.

[34] A. I. Makeev and A. V. Svidzinskii, Teor. Mat. Fiz. 44, 85 (1980) [Theor. Math. Phys. 44, 617 (1980)]; A. D. Zaikin and G. F. Zharkov, Fiz. Nizkikh Temp. 7, 375 (1981) [Sov. J. Low Temp. Phys. 7, 184 (1981)]. 